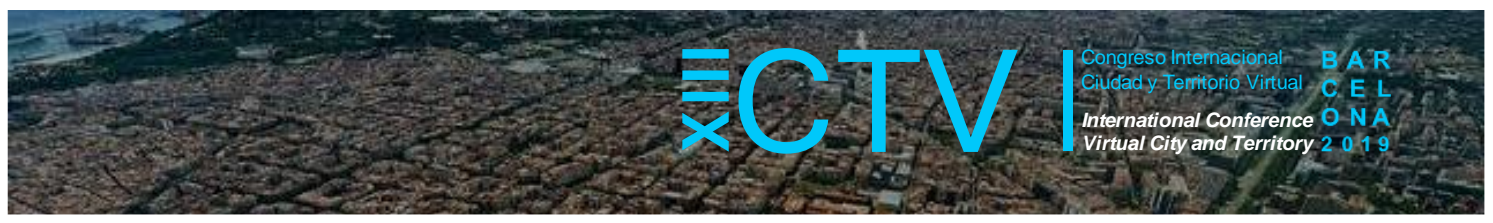

\title{
EL DERECHO A LA CIUDAD DESDE LA PERSPECTIVA DE GÉNERO EN EL BARRIO EL GALLITO (GUATEMALA)
}

\author{
Caravantes López de Lerma, Glòria Maria ${ }^{1 *}$
}

Remisión inicial: 2019-06-16; Remisión definitiva: 2019-10-15; Publicación: 2019-12-21

Citación: $\quad$ Caravantes, G. M. (2019). El derecho a la ciudad desde la perspectiva de género en el barrio el Gallito (Guatemala). En XIII CTV 2019 Proceedings: XIII International Conference on Virtual City and Territory: "Challenges and paradigms of the contemporary city": UPC, Barcelona, October 24,2019. Barcelona:CPSV, 2019,p. 8630.E-ISSN2604-6512.DOI http://dx.doi.org/10.5821/ctv.8630

\section{Resumen}

Las ciudades del mundo concentran poderes económicos y sociales y, por su propia idiosincrasia ofrecen las herramientas necesarias para hacer frente a los nuevos desafíos del desarrollo. No obstante, según ha manifestado recientemente Naciones Unidas (2018) el aumento de la vulnerabilidad y de la exclusión social, ha conllevado directamente a un crecimiento notable de las desigualdades entre regiones y ciudades. Además de ello, en las últimas décadas han emergido con fuerza espacios urbanos en los que los desequilibrios sociales, la escasez de recursos comunitarios, el deterioro y degradación del entorno, se han visto acentuados dentro de las ciudades dando lugar a los denominados barrios vulnerables. El reconocimiento de tales situaciones por parte de Naciones Unidas se ha materializado en el Objetivo de Desarrollo Sostenible 11 Ciudades y Comunidades Sostenibles al objeto de "lograr que las ciudades y los asentamientos humanos sean inclusivos, seguros, resilientes y sostenibles". La emergencia cada vez más evidente de este tipo de entornos, recupera necesariamente la concepción teórica del derecho a la ciudad ya que: "no puede concebirse como un simple derecho de visita o retorno hacia las ciudades tradicionales. Sólo puede formularse como derecho a la vida urbana, transformada, renovada" (Lefebvre, 1969, p. 138).

Por todo ello, el estudio que se presenta está centrado en la ciudad de Guatemala cuya selección se ha basado en la accesibilidad a contactos directos con profesionales que trabajan en la ciudad y que, gracias a las personas contactadas, ha sido posible establecer más contactos con la ciudadanía residente en el barrio El Gallito, ubicado en la ciudad capital. En la última Conferencia sobre Vivienda y el Desarrollo Urbano Sostenible (2016), la propia intervención de la Delegación de Guatemala manifestó ser el país menos urbanizado de la región más urbanizada del mundo y destacó la oportunidad de redefinir el modelo de desarrollo del país, enmarcando en dicho contexto la Agenda Urbana GT de carácter nacional. De este modo, se destaca la necesidad de una planificación urbana con la finalidad de reducir las brechas de la desigualdad y evitar la extensión de áreas urbanas con personas en zonas de alto riesgo, entre otras. No obstante, cabe señalar que Guatemala es uno de los catorce países con mayores índices de violencia del mundo, agravado por la presencia de grupos delincuenciales. La presencia de maras y de los banderas en diferentes zonas de Guatemala y de diferentes países de Latinoamérica, forman parte del día a día de la ciudadanía residente en estos entornos. De este modo, el objetivo se corresponde con "determinar las diferentes tipologías de sociabilidad existentes en el entorno barrial de El Gallito (Guatemala) desde la perspectiva comunitaria". Para la consecución de dicho objetivo, se han realizado un total diez audiciones (individuales y grupales) con la finalidad de discernir cómo es la vida urbana en El Gallito por parte de diferentes perfiles: técnicos, religiosos y ciudadanos, y en especial, qué papel juegan las mujeres en el quehacer urbano de El Gallito. Para el análisis de las distintas audiciones, se ha empleado el programa informático Maxqda2018 con la finalidad de codificar y categorizar los discursos de las personas audicionadas en función del objetivo e indicadores de la presente comunicación. En lo relativo a los resultados, cabe destacar que han sido obtenidos en el marco de una investigación realizada a nivel internacional con la finalidad de analizar el fenómeno de la vulnerabilidad y la exclusión social en Guatemala y en España a partir de la selección de dos barrios vulnerables. En este caso, entre los principales resultados destaca la violencia (ciudadana e institucional) a la que se encuentran sometidos sus residentes, con mención especial a las adolescentes y a las mujeres de El Gallito. Además de ello, el desarrollo de la capacidad de resiliencia territorial como medio para la convivencia frente al clima de violencia de los grupos delincuenciales y bandas organizadas, es una de las cuestiones clave que indirectamente ha sido mencionada a lo largo de los discursos. Sumado al clima de estigmatización y rechazo poblacional a las personas residentes en El Gallito, dan como resultado la identificación de este barrio como un espacio vulnerable de la ciudad capital.

\footnotetext{
1 Trabajadora social y Máster en Cooperación Internacional al Desarrollo, Universitat de Valencia (UPV), https://orcid.org/0000-0003-2656-7032. *Correo de contacto: gloriamariacaravantes@gmail.com
} 


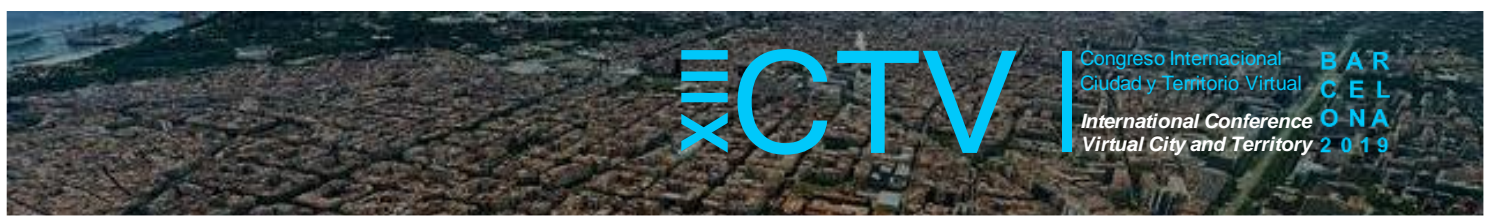

Por último, entre las conclusiones más destacadas del estudio cabe señalar, por un lado, la parálisis de la administración ante la cronicidad de la situación, y, por otro lado, la existencia de fenómenos explícitos e implícitos a la comunidad como sucede con la fuerte estigmatización social hacia el barrio y sus residentes, así como la naturalización (institucional y ciudadana) del control ejercido por los grupos y bandas organizadas sobre los límites geográficos de $\mathrm{El}$ Gallito.

\section{Abstract}

The cities of the world concentrate economic and social powers and, by their own idiosyncrasy, offer the necessary tools to face the new development challenges. However, as recently stated by the United Nations (2018), the increase in vulnerability and social exclusion has directly led to a notable growth in inequalities between regions and cities. In addition, in recent decades' urban spaces have emerged strongly in which social imbalances, lack of community resources, deterioration and degradation of the environment, have been accentuated within cities giving rise to so-called vulnerable neighborhoods. The recognition of such situations by the United Nations has materialized in the Sustainable Development Goal 11 Sustainable Cities and Communities in order to "make cities and human settlements inclusive, safe, resilient and sustainable." The increasingly evident emergence of this type of environment necessarily recovers the theoretical conception of the right to the city since: "it cannot be conceived as a simple right of visit or return to traditional cities. It can only be formulated as the right to urban life, transformed, renewed" (Lefebvre, 1969, p. 138).

Therefore, the study presented is focused on the city of Guatemala whose selection has been based on accessibility to direct contacts with professionals working in the city and that, thanks to the people contacted, it has been possible to establish more contacts with citizenship resident in the El Gallito neighborhood, located in the capital city. In the last Conference on Housing and Sustainable Urban Development (2016), the intervention of the Delegation of Guatemala declared itself to be the least urbanized country in the most urbanized region in the world and highlighted the opportunity to redefine the country's development model, framing in this context the Urban Agenda GT of national character. In this way, the need for urban planning is highlighted in order to reduce inequality gaps and avoid the extension of urban areas with people in high-risk areas, among others. However, it should be noted that Guatemala is one of the fourteen countries with the highest rates of violence in the world, aggravated by the presence of criminal groups. The presence of maras and flags in different areas of Guatemala and different countries of Latin America, are part of the day-to-day life of citizens residing in these environments. Thus, the object corresponds to "determine the different types of sociability existing in the neighborhood of El Gallito (Guatemala) from the community perspective." To achieve this goal, a total of ten auditions (individual and group) have been made in order to discern what urban life is like in El Gallito by different profiles: technicians, religious and citizens, and in particular, what role they play Women in the urban work of El Gallito. For the analysis of the different auditions, the Maxqda2018 computer program has been used to codify and categorize the speeches of the auditioned people according to the objective and indicators of this communication. Regarding the results, it should be noted that they have been obtained within the framework of an investigation carried out at the international level in order to analyze the phenomenon of vulnerability and social exclusion in Guatemala and in Spain from the selection of two neighborhoods vulnerable. In this case, the main results include the violence (citizen and institutional) to which its residents are subjected, with special mention to adolescents and women of El Gallito. In addition, the development of the capacity of territorial resilience as a means of coexistence in the face of the climate of violence of criminal groups and organized gangs, is one of the key issues that has been indirectly mentioned throughout the speeches. In addition to the stigmatization climate and population rejection of people living in El Gallito, they result in the identification of this neighborhood as a vulnerable space in the capital city.

Finally, among the most outstanding conclusions of the study it is worth pointing out, on the one hand, the paralysis of the administration due to the chronicity of the situation, and, on the other hand, the existence of explicit and implicit phenomena to the community, as is the case with the strong stigmatization social towards the neighborhood and its residents, as well as the naturalization (institutional and citizen) of the control exercised by groups and bands organized over the geographical boundaries of El Gallito.

Palabras Clave: Barrios vulnerables; perspectiva de género; Guatemala; sociabilidad

Key words: Vulnerable neighborhood; gender perspective; Guatemala; sociability

\section{Introducción}

Las desigualdades y los desequilibrios territoriales se han ido incrementando progresivamente en las últimas décadas como consecuencia de distintos factores: cambio climático, 


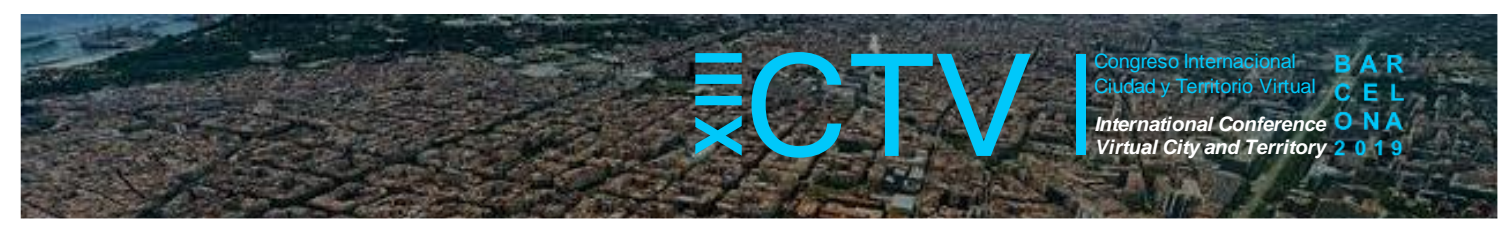

vulnerabilidad y/o exclusión social, polarización del crecimiento económico... Tal y como señala Naciones Unidas (2018, p. 3): "detener y corregir el aumento de la desigualdad también es vital para que en adelante el crecimiento sea equilibrado y sostenible".

En el espacio urbano, convergen los efectos y consecuencias de tales desigualdades, especialmente en aquellas áreas vulnerables de nuestras ciudades. Según ONU-Hábitat, el número de asentamientos precarios ${ }^{2}$ se ha ido incrementando en las ciudades y en este sentido, se han llevado a cabo actuaciones institucionales tales como el Programa Participativo de Mejoramiento de Barrios Precarios, al objeto de mejorar la calidad residencial y las condiciones vitales de las personas residentes en este tipo de entornos. El crecimiento de la vulnerabilidad en las ciudades no es una cuestión baladí. Con los Objetivos de Desarrollo Sostenible (2015), Naciones Unidas incorporó en la enunciación de los 17 objetivos, mención especial a las ciudades con el ODS 11 sobre Ciudades y Comunidades Sostenibles, con la meta de "lograr que las ciudades y los asentamientos humanos sean inclusivos, seguros, resilientes y sostenibles".

Por ello, la emergencia cada vez más evidente de este tipo de entornos, recupera necesariamente la concepción teórica del derecho a la ciudad ya que: "no puede concebirse como un simple derecho de visita o retorno hacia las ciudades tradicionales. Sólo puede formularse como derecho a la vida urbana, transformada, renovada" (Lefebvre, 1969, p. 138). En el caso de la situación de las mujeres en las ciudades, del mismo modo que sucede en el ámbito laboral, familiar, doméstico, educativo... se encuentran en posición de inferioridad. "Las desigualdades de género persisten en muchos países y contextos (menores índices de escolarización secundaria, el acceso a un empleo decente, la representación política y la naturaleza de género de la pandemia del VIH" (ONU-Hábitat, 2016, p. 1).

En el último informe sobre Ciudades Seguras y Espacios Públicos Seguros (2017) de ONUMujeres $^{3}$, se destaca que la lucha por la igualdad entre mujeres y hombres es un objetivo para el desarrollo urbano en conjunto de carácter sostenible e inclusivo con la totalidad de la ciudadanía. Combatir la normalización del acoso sexual en los espacios públicos y privados, la inseguridad urbana, y las desigualdades salariales por razón de género, entre otras cuestiones, supone por un lado combatir las situaciones de vulnerabilidad de mujeres y niñas; y, por otro lado, ampliar las oportunidades de desarrollo humano para todas y todos. En el caso que nos ocupa, se presentan parte de los resultados obtenidos en la investigación "El análisis de la vulnerabilidad y la exclusión social en el Sur y en el Norte: un estudio de casos comparado a partir de el barrio El Gallito (Guatemala) y el barrio de La Coma (España)" Caravantes et al., (2018). De este modo, el objeto de la presente comunicación se corresponde con "determinar las diferentes tipologías de sociabilidad del entorno urbano de El Gallito (Guatemala) a partir de la perspectiva de género".

Para discernir entre las diferentes tipologías de sociabilidad que tienen lugar en el contexto objeto de estudio, se ha tomado como punto de partida la teoría acuñada por Carlos Giménez

\footnotetext{
2 Según la definición de ONU - Hábitat (2017), los 'asentamientos precarios' o 'barrios precarios' se caracterizan por: acceso inadecuado a agua potable, acceso inadecuado a infraestructura y saneamiento, calidad estructural reducida en la vivienda, superpoblación y estatus residencial precario.

3 "Organización de las Naciones Unidas dedicada a promover la igualdad de género y el empoderamiento de las mujeres como defensora mundial de mujeres y niñas" (ONU - Mujeres, 2017).
} 


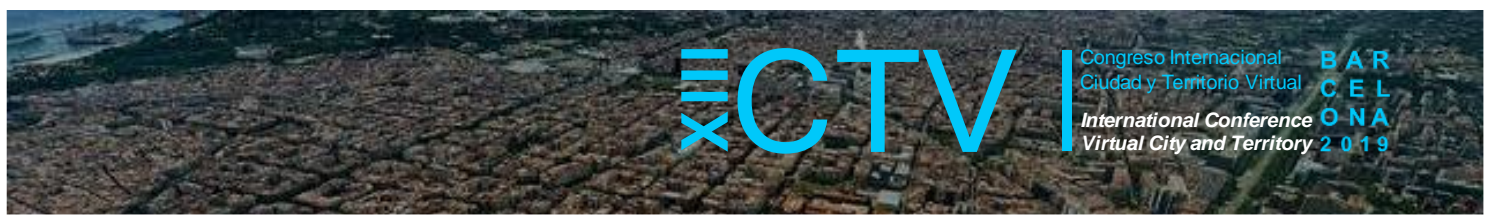

(2011). De acuerdo con el autor, a fin de dirimir el "estado de la convivencia" (Giménez, 2015) en barrios con una diversidad sociocultural elevada, se distinguen las siguientes tres tipologías:

1) Convivencia: relación pacífica donde los conflictos son abordados mediante el diálogo y el respeto. Asimismo, esta tipología implica una cordial relación entre los diferentes agentes comunitarios (administración pública, recursos técnicos - públicos y privados -, y sociedad civil organizada y ciudadanía en su conjunto, dado que comparten un mismo espacio y tiempo.

2) Coexistencia: a diferencia de la convivencia, ésta no implica, en términos generales, una situación considerada como negativa. El modelo de sociabilidad enmarcada en esta tipología, está condicionada por una relación de carácter pasivo entre los agentes comunitarios.

3) Hostilidad: esta categoría implica el polo opuesto al modelo de convivencia. La hostilidad se caracteriza fundamentalmente por relaciones fundadas en la animadversión, recelo, odio, "violencia, latente o manifiesta, estructural, física o simbólica" (Galtung, 1964) (cit. en Giménez, 2015, p. 52).

En base a ello, para cada una de las categorías se han acuñado las siguientes dimensiones: relacionales, actitudinales, normativos, axiológicos, participativos, comunicaciones, conflictuales, identitarios y políticos (Giménez, 2011; 2007; 2005). Sin embargo, a lo largo de la presente comunicación nos basaremos en las siguientes dimensiones para discernir los distintos tipos de sociabilidad.

Tabla 1. Tipologías de sociabilidad

\begin{tabular}{|c|c|c|c|c|}
\hline & & CONVIVENCIA & COEXISTENCIA & HOSTILIDAD \\
\hline \multirow{5}{*}{ 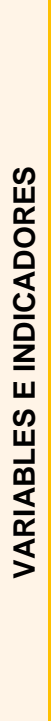 } & RELACIONAL & $\begin{array}{l}\text { Relaciones activas } \\
\text { de reciprocidad. } \\
\text { Vínculos sólidos. }\end{array}$ & Relaciones no activas. & $\begin{array}{l}\text { Relaciones de } \\
\text { desconfianza, } \\
\text { animadversión, evitación... }\end{array}$ \\
\hline & NORMATIVA & $\begin{array}{l}\text { Respeto a las } \\
\text { normas jurídicas y } \\
\text { consuetudinarias. }\end{array}$ & $\begin{array}{l}\text { Nivel mínimo de } \\
\text { cumplimiento de las } \\
\text { normas básicas. }\end{array}$ & $\begin{array}{l}\text { No cumplimiento de normas } \\
\text { democráticas. }\end{array}$ \\
\hline & AXIOLÓGICA & $\begin{array}{l}\text { Respeto a los } \\
\text { valores de la } \\
\text { comunidad. }\end{array}$ & $\begin{array}{l}\text { Aceptación relativa de } \\
\text { valores divergentes. }\end{array}$ & $\begin{array}{l}\text { Valores básicos no } \\
\text { compartidos. }\end{array}$ \\
\hline & CONFLICTUAL & $\begin{array}{l}\text { Conflictos } \\
\text { abordados de forma } \\
\text { dialogada. } \\
\text { Negociación y } \\
\text { mediación. }\end{array}$ & $\begin{array}{l}\text { Mera inexistencia de } \\
\text { conflictos degenerados } \\
\text { en disputas. } \\
\text { Paz pasiva. }\end{array}$ & $\begin{array}{l}\text { Conflictos latentes no } \\
\text { dialogados (conflicto } \\
\text { manifiesto). } \\
\text { No hay paz social. }\end{array}$ \\
\hline & ACTITUDINAL & $\begin{array}{l}\text { Aceptación plena de } \\
\text { la diversidad. }\end{array}$ & $\begin{array}{l}\text { Respeto pasivo } \\
\text { Tolerancia "de la } \\
\text { resistencia." }\end{array}$ & $\begin{array}{l}\text { No hay respeto ni tolerancia } \\
\text { Actitud agresiva, agresión } \\
\text { verbal y física. }\end{array}$ \\
\hline
\end{tabular}

Fuente: Elaboración propia a partir de Giménez (2011).

A partir de estas cinco variables e indicadores para cada una de ellas, se analizarán las distintas tipologías de sociabilidad en el barrio de El Gallito. 


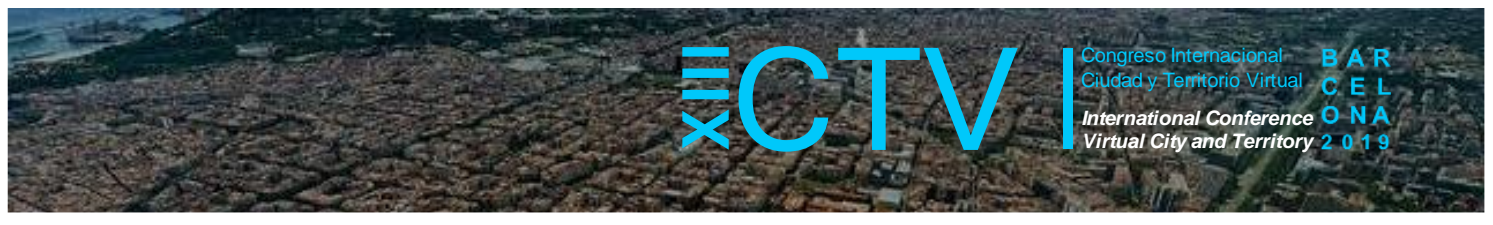

\subsection{Contextualización del objeto de estudio}

De acuerdo con lo señalado previamente, los resultados expuestos a lo largo de las siguientes páginas proceden de un estudio previo realizado a nivel internacional. En el caso que nos ocupa, la selección de Guatemala se ha caracterizado por su ubicación en el Sur y por la disponibilidad de contacto directo con agentes comunitarios (ciudadanía y recursos técnicos). Además de ello, de acuerdo con la Conferencia de Vivienda y Desarrollo Humano Sostenible (2016) Guatemala es el país menos urbanizado de la región más urbanizada del mundo y es uno de los catorce países con mayor índice de violencia del mundo.

Entre algunos datos característicos de Guatemala, según los últimos informes del Programa de Naciones Unidas para el Desarrollo (PNUD) cabe destacar que dispone de un Índice de Desarrollo Humano (IDH) medio $(0,560)$. No obstante, si consideramos el Índice de Desarrollo de Género (IDG) podemos observar en la Tabla 2 cómo se ha reducido en casi tres puntos del año $2010(0,713)$ al $2018(0,494)$ (Caravantes, 2019).

Asimismo, contemplando otras cifras de carácter nacional, la progresión de Guatemala respecto a la desigualdad existente en materia educativa y económica para el período 2010 2018 se ha caracterizado por un aumento en ambos campos tal y como se detalla a continuación.

Tabla 2. Datos macro sobre Guatemala ${ }^{4}$

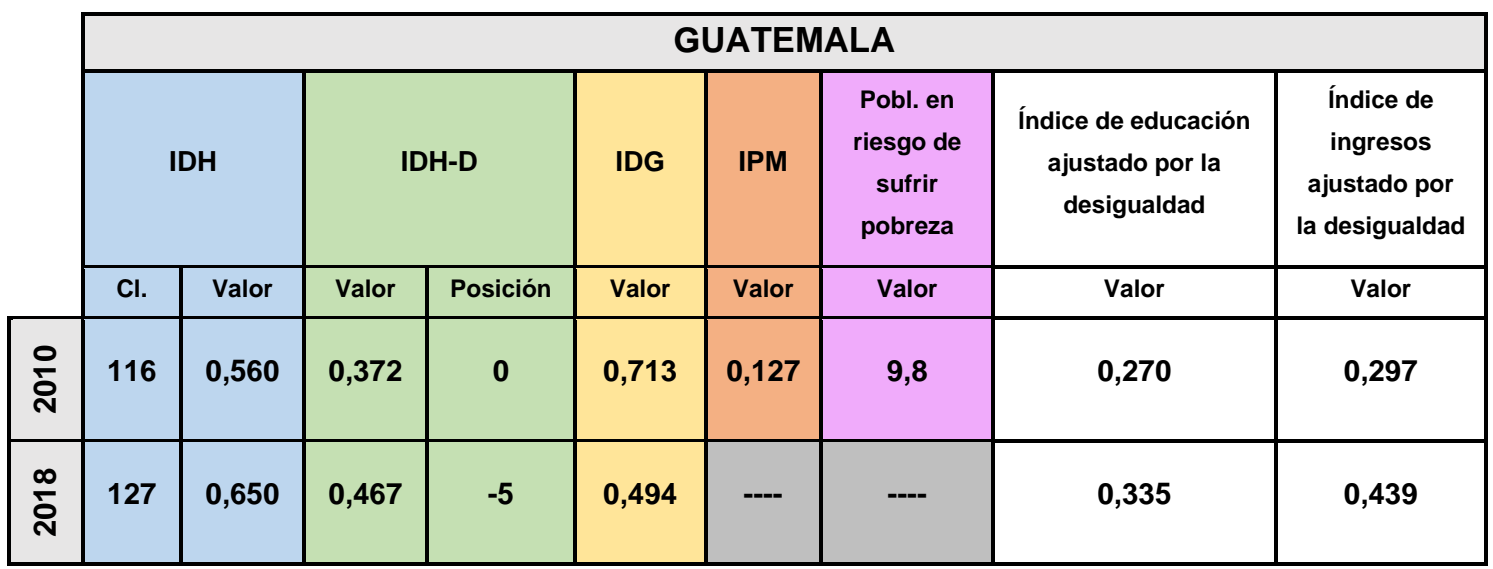

Fuente: Datos extraídos a partir del Informe de Desarrollo Humano del PNUD (2010; 2018)

Por su parte, producto del planeamiento urbano de la ciudad, Guatemala consta de 25 zonas ordenadas en sentido contrario a las agujas del reloj en forma circular. En el periodo de 19261930, el gobierno del presidente de la República Lázaro Chacón tomó la decisión de formar barrios para obreros. En el proceso de adjudicación de las parcelas y la transferencia de terrenos, se previó la inversión en la formación de las estructuras necesarias para la provisión del agua potable a la población residente en El Gallito. Posteriormente a la década de los años 30, se llevaron a cabo la instalación de drenajes, pavimentación, la introducción del agua potable y el mantenimiento de los servicios existentes (Zea, 2013).

\footnotetext{
${ }^{4}$ Abreviaturas de la Tabla 2: IDH: Índice de Desarrollo Humano, IDH-D: Índice de Desarrollo Humano ajustado por la Desigualdad, IDG: Índice de Desarrollo de Género, IPM: Índice de Pobreza Multidimensional.
} 


\section{Mapa 1. Situación geográfica de la ciudad de Guatemala}

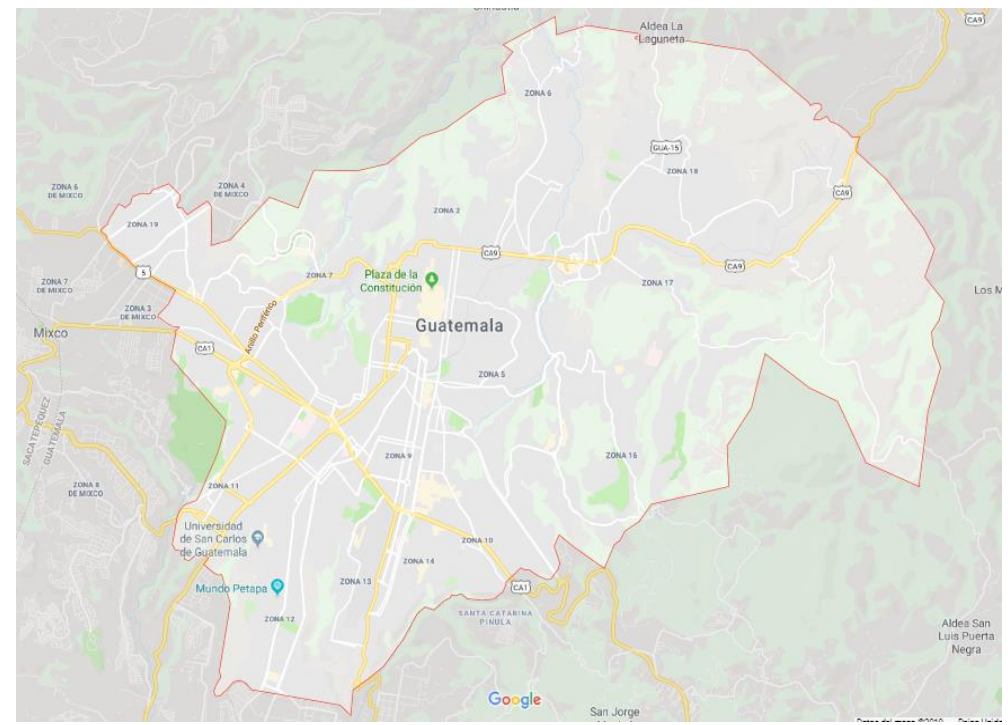

Fuente: Google Maps.

La ubicación geográfica del barrio de El Gallito, se encuentra en el área central de la ciudad capital, concretamente en la Zona $3^{5}$ cuya zona cuenta con un total de 25.501 habitantes (Secretaría de Seguridad Alimentaria y Nutricional, 2009). ${ }^{6}$

Mapa 2. Delimitación territorial del barrio de El Gallito

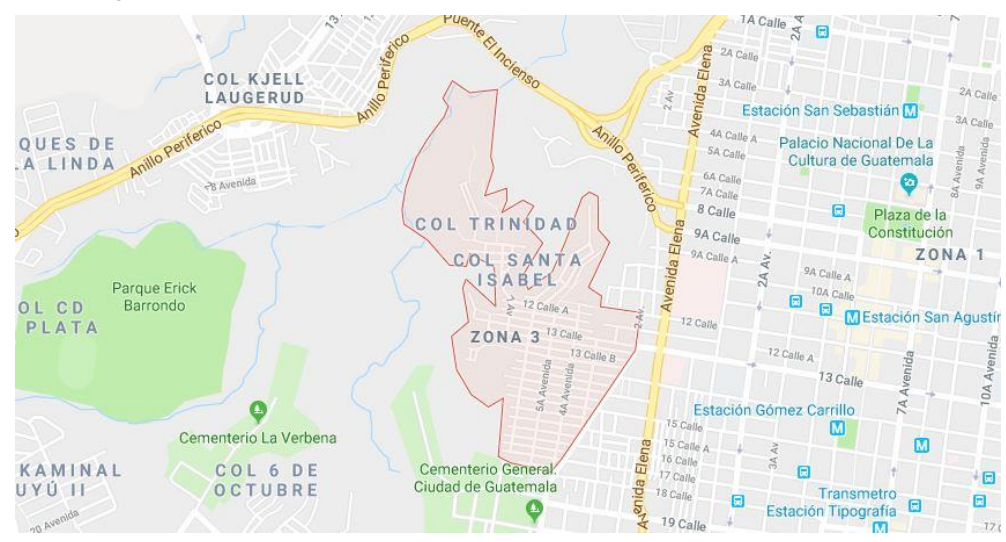

Fuente: Google Maps.

En cuanto a las limitaciones y características físicas, cabe señalar que ha habido un cambio considerable en los últimos 70 años. En los años 40, época donde se sitúa su creación, había 17 entradas que daban acceso al barrio, pero posteriormente, en la década de los 90, con la llegada de grupos delincuenciales y traficantes, se volvió un barrio peligroso:

\footnotetext{
${ }^{5}$ En el periodo de 1926-1930, el gobierno del presidente de la República Lázaro Chacón tomó la decisión de formar barrios para obreros. En el proceso de adjudicación de las parcelas y la transferencia de terrenos, se previó la inversión en la formación de las estructuras necesarias para la provisión del agua potable a la población residente en El Gallito. Posteriormente a la década de los años 30 , se llevaron a cabo la instalación de drenajes, pavimentación, la introducción del agua potable y el mantenimiento de los servicios existentes (Zea, 2013).

6 Últimos datos disponibles.
} 


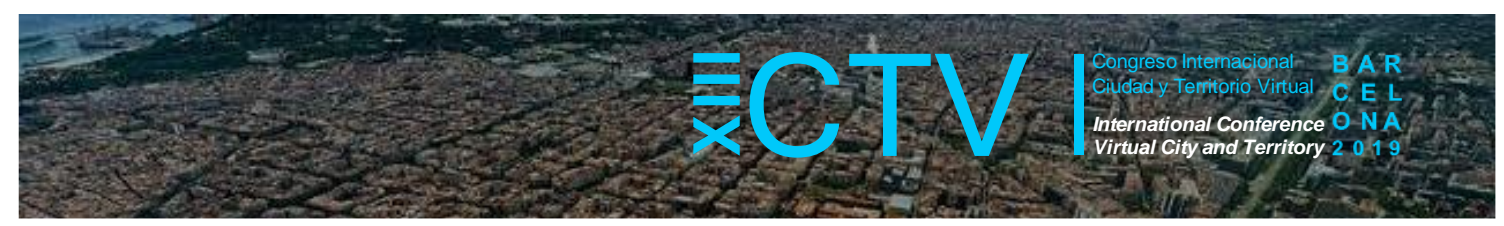

"Fue catalogado como barrio rojo por la policía, dada la peligrosidad que existe allá. (...) Hubo un momento en el que la policía dejó 3 ó 4 entradas para controlar un poco más el acceso de las personas al barrio con la finalidad de que volviera a ser lo que era antes, digamos que fue una especie de cierre parcial a vehículos y a las personas. Ello se volvió peor, porque la policía no podía entrar, se volvió como una especie de lugar sacrosanto y toda la gente que vivía allí se quedó como aislada" (AGTS) ${ }^{7}$.

Por otra parte, en cuanto a la configuración interna del barrio, se ha señalado una notable diferencia respecto a la Zona Alta y Baja del mismo. La Zona Alta es aquella que se encuentra más próxima al centro de la ciudad (Zona 1), mientras que la Zona Baja hace referencia a la zona colindante con el barranco (Zona 7).

Sus límites territoriales están marcados de la siguiente manera: al Norte 12 calle y 3 avenida, 13 calle y avenida de El Cementerio y los asentamientos 3 de Mayo, La JOYA, Progreso, San Francisco, Colonia Santa Isabel, El Bosque, El Recuerdo, Unidad y Progreso y Las Calaveras, al Este por la Avenida de El Cementerio y la 3 avenida, al Sur por el Mercado de Flores y el Cementerio General, al Oeste por los asentamientos: Esfuerzo I y II Pasaje San Jorge, La Isla, La cruz, San José Buena Vista y el Relleno Sanitario (Morales Duarte, 2009, p. 21).

\section{Metodología}

Para el desarrollo de la investigación, se ha partido del estudio de caso que, en la línea de Castro $(2010$, p. 36) refiere el estudio "de un fenómeno contemporáneo dentro de su contexto real, cuando las fronteras entre el fenómeno y el contexto no son evidentes, y en la que se utilizan múltiples fuentes de evidencia".

Para la consecución del objetivo propuesto, se ha empleado la técnica cualitativa de la audición dirigida a agentes comunitarios tanto del ámbito ciudadano como del ámbito técnico y profesional, contemplando diversidad de perfiles en cuanto a años de residencia, conocimiento acerca del barrio, edad, capacidad de movilización... A continuación, se detallan específicamente los criterios de selección y los distintos perfiles de las personas informantes en el barrio de El Gallito:

Tabla 3. Perfil de las personas informantes en el barrio de El Gallito

\begin{tabular}{|c|c|c|c|}
\hline & CRITERIOS DE SELECCIÓN & $\begin{array}{l}\text { BARRIO EL } \\
\text { GALLITO }\end{array}$ & CÓDIGO \\
\hline \multirow{3}{*}{ 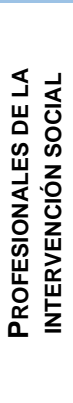 } & $\begin{array}{l}\text { - } \quad \text { Conocimiento del barrio } \\
\text { - Edad: } 48,49 \text { y } 50 \\
\text { - } \quad \text { Experiencia profesional en los barrios más de } 4 \text { años } \\
\text { - } \quad \text { ontacto directo con la población residente }\end{array}$ & TRABAJADOR SOCIAL & AGTS \\
\hline & $\begin{array}{l}\text { - } \quad \text { Conocimiento del barrio } \\
\text { - } \quad \text { Edad: } 25 \text { y } 31 \\
\text { - } \quad \text { Contacto directo con la población residente }\end{array}$ & TÉCNICA MUNICIPAL & AGTM \\
\hline & $\begin{array}{l}\text { - } \text { Conocimiento del barrio } \\
\text { - } \quad \text { Edad: } 29 \text { y } 39 \\
\text { Realización de investigaciones en profundidad sobre los } \\
\text { barrios objeto de estudio. } \\
\text { - Experiencia profesional en el municipio }\end{array}$ & EXPERTO & AGE \\
\hline
\end{tabular}

\footnotetext{
${ }^{7}$ Audición realizada al/la trabajador/a social del barrio.
} 


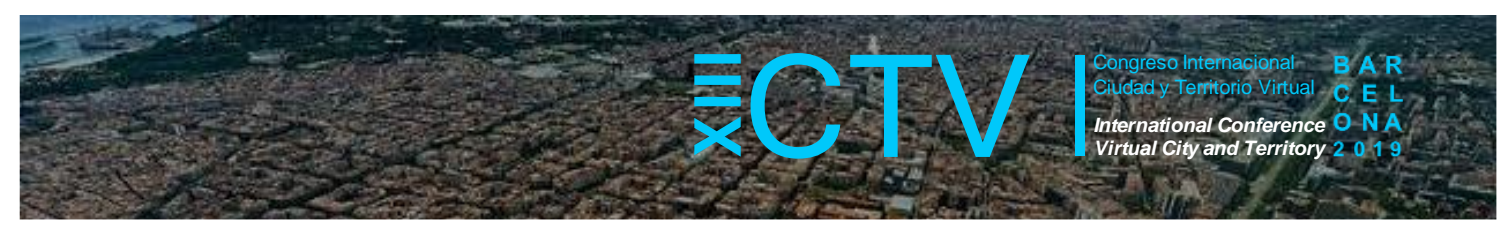

\begin{tabular}{|c|c|c|c|}
\hline & CRITERIOS DE SELECCIÓN & $\begin{array}{l}\text { BARRIO EL } \\
\text { GALLITO }\end{array}$ & CÓDIGO \\
\hline \multirow{7}{*}{ 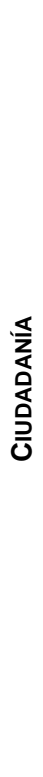 } & $\begin{array}{l}\text { - Conocimiento } \\
\text { - } \text { Pesencia en el barrio } \\
\text { - Capacionte de reconocidad de movilización social } \\
\text { Cocial }\end{array}$ & IGLESIA EVANGÉLICA & AGIE \\
\hline & $\begin{array}{l}\text { - } \quad \text { Conocimiento del barrio } \\
\text { - } \quad \text { Reconcia en el barrio } \\
\text { - } \quad \text { Capacidad de movilización social }\end{array}$ & $\begin{array}{l}\text { HERMANDAD EL } \\
\text { GALLITO }\end{array}$ & AGIC \\
\hline & $\begin{array}{l}\text { - Conocimiento del barrio } \\
\text { - } \text { Resad: } 20 \\
\text { - Varón }\end{array}$ & VECINO JOVEN & AGV01 \\
\hline & $\begin{array}{l}\text { - } \quad \text { Conocimiento del barrio } \\
\text { - } \quad \text { Residencia en el barrio más de } 19 \text { años }\end{array}$ & VECINO JOVEN & AGV02 \\
\hline & $\begin{array}{l}\text { - } \text { Conocimiento del barrio } \\
\text { Edad: } 19\end{array}$ & VECINO JOVEN & AGV03 \\
\hline & $\begin{array}{l}\text { - } \quad \text { Conocimiento del barrio } \\
\text { - Mujer }\end{array}$ & VECINA JOVEN & AGV04 \\
\hline & $\begin{array}{l}\text { - } \quad \text { Conocimiento del barrio } \\
\text { - } \quad \text { Mujesidencia en el barrio más de } 19 \text { años }\end{array}$ & VECINA JOVEN & AGV05 \\
\hline
\end{tabular}

Fuente: Elaboración propia a partir de Caravantes (2019).

La técnica de la audición está orientada hacia el conocimiento del objeto de estudio en su globalidad y la información obtenida permite contemplar la pluralidad de aspectos sobre los que se desea incidir. Asimismo, la audición parte de la premisa de que:

Hay que escuchar lo que dice y hace la gente, y las preguntas que surgen de su praxis, pero no de cualquier forma. Son muchas las verdades parciales que se construyen cotidianamente, y para ser fieles a los cambios que la gente reclama, debemos aplicar un cierto rigor que haga emerger la construcción colectiva de verdades más profundas, más transformadoras, y no tanto lo primero que se nos ocupa (Rodríguez Villasante, 2002, p. 13).

En este sentido, puede emplearse para el conocimiento, bien de una comunidad en su totalidad o bien, hacia un tema específico de la vida en comunidad. En el caso que nos concierne, la orientación de la audición se corresponde con la primera vertiente, correspondiente al conocimiento del barrio de El Gallito en su totalidad desde una perspectiva integral y contemplando las diferentes partes que conforman la realidad comunitaria de la ciudadanía. Por otra parte, como técnica de análisis y procesamiento de datos se ha empleado el programa informático Maxqda 2018, puesto que permite un tratamiento cuantitativo de datos después de una categorización de los elementos discursivos y ofrece múltiples funcionalidades como podemos observar en la pantalla.

\section{Resultados}

Para la presentación de los resultados principales, se tomará como referencia el esquema propuesto por Giménez (2011). Partiendo de la perspectiva de género, en la variable relacional cabe destacar que existe cierta hostilidad en la relación mujeres y adolescentes - grupos delincuenciales, especialmente por el clima de violencia que se vive en el barrio: 


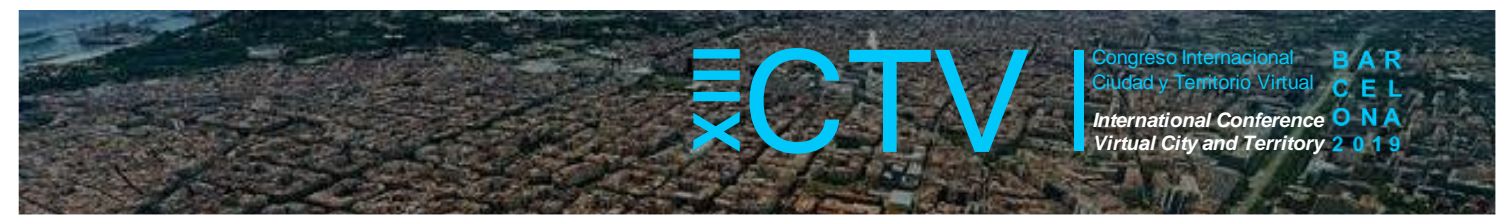

Antes era más peligroso y ahora ya no tanto. Eso es porque han ido matando a la gente con la que había más altercados en el barrio. Antes una vez al año había una balacera y se ponían a disparar entre ellos. Las pandillas se ponían a pelear por la distribución de la droga (AGV05).

Tabla 4. Resultados generales a partir de Giménez (2011)

\begin{tabular}{|c|c|c|c|c|}
\hline & & CONVIVENCIA & COEXISTENCIA & HOSTILIDAD \\
\hline \multirow{5}{*}{ 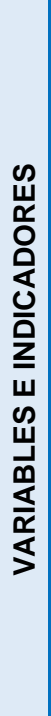 } & RELACIONAL & $\begin{array}{l}\text { Relaciones activas } \\
\text { de reciprocidad. } \\
\text { Vínculos sólidos. }\end{array}$ & Relaciones no activas. & $\begin{array}{l}\text { Relaciones de } \\
\text { desconfianza, } \\
\text { animadversión, evitación... }\end{array}$ \\
\hline & NORMATIVA & $\begin{array}{l}\text { Respeto a las } \\
\text { normas jurídicas y } \\
\text { consuetudinarias. }\end{array}$ & & \\
\hline & AXIOLÓGICA & $\begin{array}{l}\text { Respeto a los } \\
\text { valores de la } \\
\text { comunidad. }\end{array}$ & & \\
\hline & CONFLICTUAL & & $\begin{array}{l}\text { Mera inexistencia de } \\
\text { conflictos degenerados } \\
\text { en disputas. } \\
\text { Paz pasiva. }\end{array}$ & $\begin{array}{l}\text { Conflictos latentes no } \\
\text { dialogados (conflicto } \\
\text { manifiesto). } \\
\text { No hay paz social. }\end{array}$ \\
\hline & ACTITUDINAL & & $\begin{array}{l}\text { Respeto pasivo } \\
\text { Tolerancia "de la } \\
\text { resistencia." }\end{array}$ & $\begin{array}{l}\text { No hay respeto ni tolerancia } \\
\text { Actitud agresiva, agresión } \\
\text { verbal y física. }\end{array}$ \\
\hline
\end{tabular}

Fuente: Elaboración propia a partir de Giménez (2011).

No obstante, ello contrasta con la opinión de aquellos vecinos que refieren sentirse seguros gracias a la presencia de tales grupos delincuenciales:

Entró un súper carro con los vidrios polarizados y demás y entró a toda pastilla por el barrio. Yo le dije a mis primitos que se metieran en la casa porque las motos estaban detrás del coche. Cuando bajaron los vidrios, se dieron cuenta que era una familia de coreanos, les preguntaron que qué habían venido a buscar al barrio. Ellos se disculparon y les dijeron que se habían perdido. Así que el jefe de los narcos les dijo: no se preocupen, si ustedes se han perdido yo les acompaño a la entrada del barrio, pero ándense con ojo porque esto es una zona roja y es peligrosa. Ya te digo que, si hubiese sido en otro momento de épocas anteriores, habrían dejado el coche hecho lata, como decimos acá. Yo estoy tranquilo porque me conocen y no pasa nada. Todo cambia en función del jefe que haya. Por un lado, están los barrio dieciocho, los mara salvatrucha y los paisas, que estos últimos no pertenecen a ninguno de los anteriores pero extorsionan (AGV03).

Del mismo discurso, en la variable normativa y axiológica se desprende el respeto de la comunidad hacia los valores imperantes tales como el respeto hacia quienes custodian los límites del barrio. En lo que concierne a la variable conflictual, es notable la alternancia entre paz pasiva - en buena medida marcado por el respeto a los grupos delincuenciales - y la ausencia de ella, dado el continuo clima de violencia. En buena medida, ello también justifica la variable actitudinal fundamentada en la coexistencia y la hostilidad: 


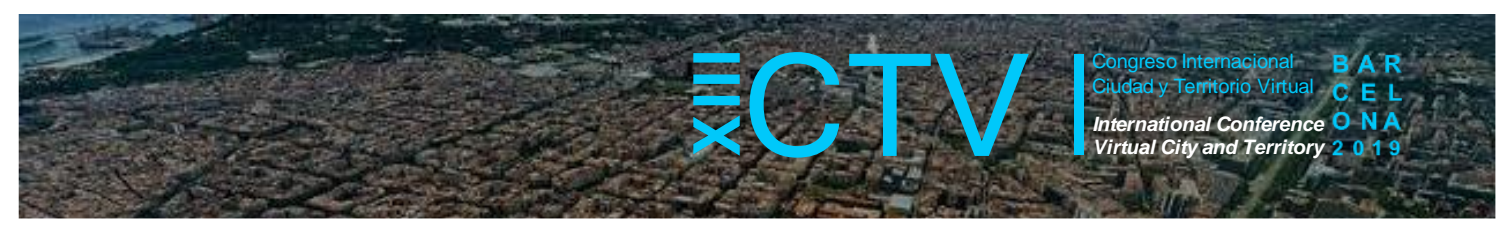

Si no bajas los vidrios polarizados te pueden perseguir en motos o les balacean directamente. (...) Antes era más peligroso y ahora ya no tanto. Eso es porque han ido matando a la gente con la que había más altercados en el barrio. Antes una vez al año había una balacera y se ponían a disparar entre ellos. Las pandillas se ponían a pelear por la distribución de la droga (AGV03).

La sensación de inseguridad ciudadana, unida a la percepción social negativa (tanto a nivel exógeno como endógeno) y el tráfico de droga, han sido los temas más señalados respecto a factores de riesgo:

\section{Gráfico 7. Factores de riesgo de El Gallito}

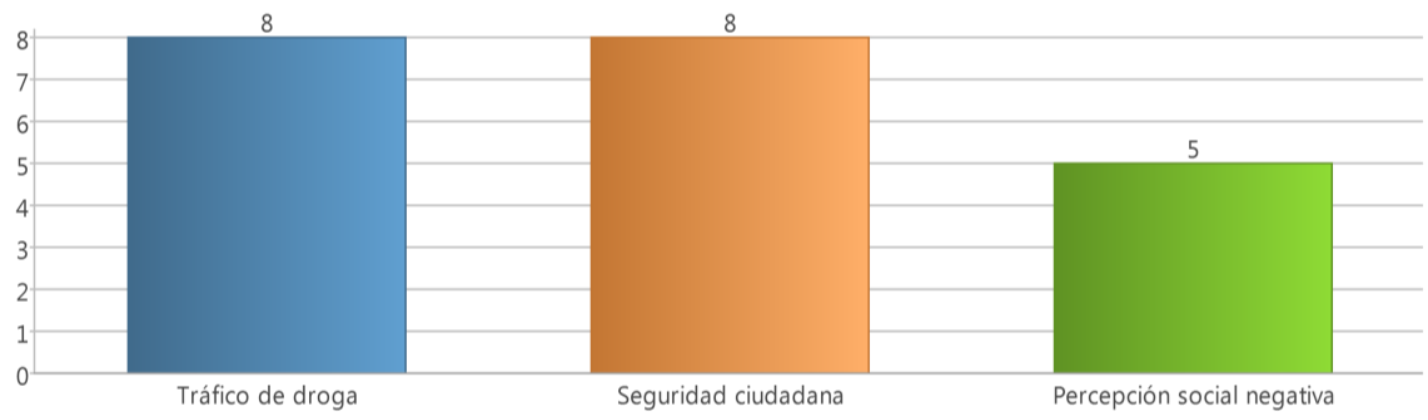

Fuente: Gráfico extraído de Maxqda 2018

En este sentido, el conjunto de variables e indicadores, así como los factores de riesgo más señalados por las personas audicionadas, implican en buena medida la alternancia entre la coexistencia y la hostilidad en lo que respecta a la tipología de sociabilidad imperante en el barrio de El Gallito.

\section{Conclusiones}

Para concluir, cabe destacar que no existe una tipología única en la que encuadrar la pluralidad de las situaciones de sociabilidad que acontecen en los barrios, dado que como se ha presentado a lo largo de los resultados, en algunas situaciones convergen dos o tres tipologías simultáneamente.

Por otra parte, marcado por la propia existencia de los grupos delincuenciales, el clima de violencia en el barrio de El Gallito se ha naturalizado por parte de la ciudadanía. Ésta ha manifestado sentirse segura en el barrio gracias a su presencia. La existencia de tales grupos es conocida por las fuerzas de seguridad, pero tal y como refieren las personas audicionadas, existe cierta permisividad respecto a la violencia que ejercen tales grupos, llegando incluso a destacar que las fuerzas de seguridad se encuentran corrompidas.

No obstante, en mitad del clima de violencia imperante en los límites del barrio, a lo largo de la determinación de las tipologías de sociabilidad, se ha identificado aquella capacidad de las personas residentes de hacer frente a las adversidades; es decir resiliencia urbana. Esta capacidad actúa como potencialidad endógena del contexto objeto de estudio y se encuentra vinculada a la 'tolerancia de la resistencia' ya que ello, se materializa en el desarrollo y articulación de estrategias de adaptación, resistencia y recuperación frente a cualquier peligro existente. 


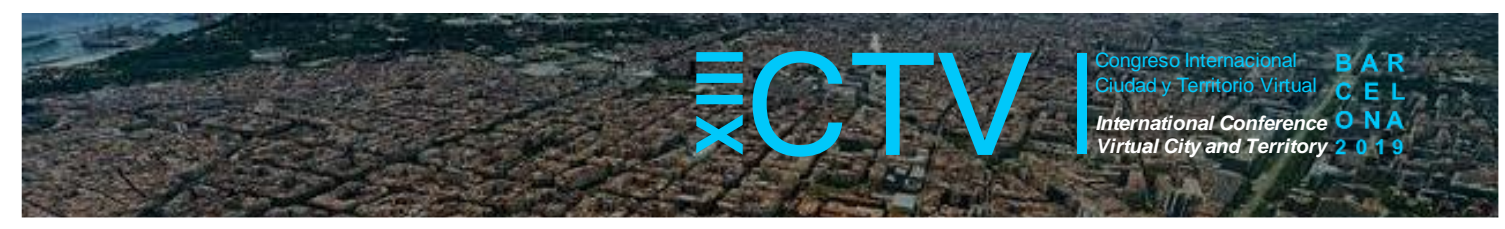

Agradecimientos: Agradecer la valentía de las mujeres que participaron desinteresadamente en la realización de esta investigación, así como a aquellos vecinos y profesionales que del mismo modo colaboraron.

Conflicto de Intereses: La autora declara que no hay conflicto de intereses.

\section{Bibliografía}

Caravantes, G. M. (2019). Barrios vulnerables: ¿derecho a la vivienda o vulnerabilidad de derechos? Una aproximación desde la exclusión residencial. Cuadernos Electrónicos de Filosofía del Derecho, 39, pp. 356-376.

Caravantes, G. M. et al. (2018). El análisis de la vulnerabilidad y la exclusión social en el Sur y en el Norte: un estudio de casos comparado a partir del barrio de El Gallito (Guatemala) y el barrio de La Coma (España). Revista DTS, pp. 47-89.

Galtung, J. (1964). Summit Meetings and International Relations, Journal of Peace Research March 1964, 1, pp. 36-54.

Giménez, C. (2015). Promoviendo la convivencia ciudadana intercultural en barrios de alta diversidad. Ideas y experiencias para una praxis comunitaria. Cuadernos Manuel Giménez Abad, 4, pp. 47-68.

Giménez, C. (2011). Convivencia e Intervención. Instituto Universitario de Investigación en Migraciones, Etnicidad y Desarrollo Social.

Giménez, C. (2007). "Migración, sociedad y cultura: la perspectiva antropológica”, en Lisón, C. (coord.). Introducción a la antropología social y cultural: teoría, método y práctica, pp. 153-190.

Giménez, C. (2005). Convivencia. Conceptualización y sugerencia para la praxis. Puntos de Vista, $\mathrm{n}^{\circ}$ 1, pp. 7-31.

ONU-Mujeres (2017). Ciudades Seguras y Espacios Públicos Seguros. Informe de Resultados Globales. Recuperado de https://bit.ly/2m48gnB

ONU-Hábitat (2016). Equidad urbana en el desarrollo: ciudades por la vida, documento conceptual. Recuperado de https://bit.ly/2lsYifs

ONU Hábitat (2015). UN-Habitat Global Activities Report. Increasing Synergy for Greater National Ownership. Recuperado de https://bit.ly/2KFrE3W

Programa de Naciones Unidas para el Desarrollo. (2010). Informe sobre Desarrollo Humano 2010. La verdadera riqueza de las naciones: caminos al desarrollo humano. Recuperado de http://bit.ly/1rXZsMx

Programa de Naciones Unidas para el Desarrollo. (2018). Informe sobre Desarrollo Humano 2016. Índices e indicadores de desarrollo humano. Recuperado de https://bit.ly/2R0FEoE

Rodríguez Villasante, T. (2002): Construyendo ciudadanía. Madrid, El Viejo Topo.

Secretaría de Seguridad Alimentaria y Nutricional (2009). Guatemala: perfiles de medios de vida. Disponible en https://bit.ly/2svANTj 